\title{
Repeated Electrical Stimulation of Reward-Related Brain Regions Affects Cocaine But Not "Natural” Reinforcement
}

\author{
Dino Levy, ${ }^{1 \star}$ Maytal Shabat-Simon, ${ }^{1,3 *}$ Uri Shalev, ${ }^{2}$ Noam Barnea-Ygael, ${ }^{1}$ Ayelet Cooper, ${ }^{1}$ and Abraham Zangen ${ }^{1}$ \\ ${ }^{1}$ Department of Neurobiology, The Weizmann Institute of Science, Rehovot 76100, Israel, ${ }^{2}$ Department of Psychology, Concordia University, Montreal, \\ Quebec, Canada H4B 1R6, and 3Department of Physiology and Pharmacology, Tel-Aviv University, Tel-Aviv 69978, Israel
}

\begin{abstract}
Drug addiction is associated with long-lasting neuronal adaptations including alterations in dopamine and glutamate receptors in the brain reward system. Treatment strategies for cocaine addiction and especially the prevention of craving and relapse are limited, and their effectiveness is still questionable. We hypothesized that repeated stimulation of the brain reward system can induce localized neuronal adaptations that may either potentiate or reduce addictive behaviors. The present study was designed to test how repeated interference with the brain reward system using localized electrical stimulation of the medial forebrain bundle at the lateral hypothalamus (LH) or the prefrontal cortex (PFC) affects cocaine addiction-associated behaviors and some of the neuronal adaptations induced by repeated exposure to cocaine. Repeated high-frequency stimulation in either site influenced cocaine, but not sucrose reward-related behaviors. Stimulation of the LH reduced cue-induced seeking behavior, whereas stimulation of the PFC reduced both cocaine-seeking behavior and the motivation for its consumption. The behavioral findings were accompanied by glutamate receptor subtype alterations in the nucleus accumbens and the ventral tegmental area, both key structures of the reward system. It is therefore suggested that repeated electrical stimulation of the PFC can become a novel strategy for treating addiction.
\end{abstract}

Key words: cocaine; addiction; intracranial electrical stimulation; self-administration; lateral hypothalamus; prefrontal cortex

\section{Introduction}

Drug addiction is a brain disease (Leshner, 1997) that causes heavy burden on society. Most advances in treating addiction address the physical dependence and withdrawal aspects that accompany it (Nestler, 2002), whereas treatments for drug craving and relapse are less established. Treatment approaches are often pharmacological, focusing on the attempt to block the drug effects, mimic their actions, or even block the addiction process (Hyman and Malenka, 2001; Nestler, 2001). Although some of these approaches showed promising results in animal studies, their potential use in humans are often limited because of toxicity, various side effects (including mood alterations), and the possibility to generalize the specific treatment to other drugs of abuse (Nestler, 2002).

Repeated drug exposure produces long-lasting neuronal adaptations (Hyman et al., 2006) in reward-related brain areas such as the nucleus accumbens (NAc), ventral tegmental area (VTA), and prefrontal cortex (PFC) that are mediated by repeated hyperactivation of the dopaminergic mesocorticolimbic pathway (Vanderschuren and Kalivas, 2000; Chefer and Shippenberg, 2002) and by glutamate transmission (Wolf et al., 2004). Glutamate transmission in these brain areas mediates relapse to

\footnotetext{
Received May 21, 2007; revised 0ct. 31, 2007; accepted 0ct. 31, 2007.

This work was supported by the Rosenzweig-Coopersmith Foundation and by the Joseph and Celia Reskin fund ${ }^{*}$ D.L. and M.S.-S. contributed equally to this work.

Correspondence should be addressed to Dr. Abraham Zangen, Department of Neurobiology, The Weizmann Institute of Science, Rehovot 76100, Israel. E-mail: a.zangen@weizmann.ac.il. DOI:10.1523/JNEUROSCI.4477-07.2007

Copyright $\odot 2007$ Society for Neuroscience 0270-6474/07/2714179-11\$15.00/0
}

cocaine-seeking behavior (Cornish and Kalivas, 2000), and there are alterations in glutamate receptor levels (Churchill et al., 1999; Sutton et al., 2003), function (Wolf, 1998), and cell surface expression (Boudreau and Wolf, 2005) in these regions after withdrawal from repeated cocaine intake. Thus, manipulations that could affect drug-induced neuroadaptations, including glutamate transmission within the reward system, that lead to reduced response to drugs or drug-associated cues, may reduce drugassociated behaviors.

A nonpharmacological candidate for inducing such effects is intracranial electrical stimulation (ICES). Stimulation of rewardrelated brain areas strongly activates the mesocorticolimbic system, causing dopaminergic elevation and increased neuronal activity in the NAc and VTA (You et al., 1998, 2001), both mediated via glutamatergic innervations (Sesack and Pickel, 1992). Therefore, repeated ICES of reward-related sites may either sensitize or desensitize responses to drugs of abuse. Indeed, ICES affects various drug-related behaviors. For example, hippocampal stimulation reinstated cocaine self-administration (Vorel et al., 2001), whereas repeated PFC stimulation sensitized cocaine-induced locomotor response (Schenk and Snow, 1994). In addition, hypothalamic intracranial self-stimulation (ICSS) delays the development of psychomotor sensitization to repeated amphetamine exposure (Wise and Munn, 1993). Finally, hypothalamic ICSS downregulates GluR1 levels in the VTA (Carlezon et al., 2001), which is opposite to the repeated cocaine exposure-induced GluR1 upregulation in the VTA (Lu et al., 2003).

We propose a novel approach for treating drug addiction using ICES in rats, which can simulate the potential effect of deep transcranial magnetic stimulation (TMS) in humans (Zangen et 
al., 2005). We hypothesized that repeated ICES of reward-related brain areas in rats pretreated with cocaine would hyperactivate the reward system and alter drug-induced neuroadaptations. Therefore, such approach could reduce cocaine reinforcement, seeking, and intake. We also compared the effects of ICES on rats self-administering cocaine to rats self-administering sucrose, which is a natural reward. Finally, we began to characterize the effects of repeated ICES on cocaine-induced neuroadaptations by measuring the expression of glutamate receptor subunits within reward-related brain sites.

\section{Materials and Methods Animals}

Male Sprague Dawley rats (Harlan, Jerusalem, Israel) weighing 250-350 g were housed in a temperature-controlled room $\left(25^{\circ} \mathrm{C}\right)$ on a regular (for locomotor activity experiments) or reversed (for self-administration experiments) $12 \mathrm{~h}$ light/dark cycle with food and water available ad libitum, unless specified otherwise. After surgery, the rats were housed individually. All experiments were conducted between 9:30 A.M. and 5:00 P.M. The study was approved by the Institutional Animal Care and Use Committee of The Weizmann Institute of Science.

\section{Drugs}

Cocaine hydrochloride (generously provided by the National Institute on Drug Abuse, National Institutes of Health) was diluted in saline $(0.9 \%$ $\mathrm{NaCl}$ ) and was injected intraperitoneally in the behavioral sensitization experiments and self-administered intravenously in the selfadministration experiments. Sucrose (J.T. Baker, Deventer, Holland) was diluted in tap water $(10 \% \mathrm{w} / \mathrm{v})$.

\section{Electrode implantation}

Rats were deeply anesthetized with ketamine $(170 \mathrm{mg} / \mathrm{kg})$ and acepromazine $(1.7 \mathrm{mg} / \mathrm{kg}$; i.p. $)$ and placed in a stereotaxic frame. Bilateral stimulating electrodes were directed toward the lateral hypothalamus ( $\mathrm{LH})$ [mediolateral $(\mathrm{ML})=1.8 \mathrm{~mm}$; dorsoventral $(\mathrm{DV})=8.5 \mathrm{~mm}$; anteroposterior $(\mathrm{AP})=-2.5 \mathrm{~mm}$ from bregma] or the PFC $(\mathrm{ML}=0.95 \mathrm{~mm}$; $\mathrm{DV}=3.4 \mathrm{~mm} ; \mathrm{AP}=+3.2 \mathrm{~mm}$ from bregma, at a lateral angle of $\left.16^{\circ}\right)$. The coordinates were taken from a rat brain atlas (Paxinos and Watson, 1998). For each pair of electrodes (Plastics One, Roanoke, VA), a miniature plastic connector held the upper end, which was attached to the skull with mini-screws and dental cement. The connector has two wires serving as ground and stimulating leads $(0.2 \mathrm{~mm}$ diameter). The stimulating lead was lowered to the appropriate brain location. The ground electrode was connected to mini-screws attached to the skull. After surgery, rats were allowed to recover for 3-6 d before being subjected to behavioral experiments. During the recovery period, the rats were handled daily.

\section{Catheter implantation}

For the cocaine self-administration experiments, a SILASTIC catheter was inserted into the rat's right jugular vein during the surgical procedure for electrode implantation. The catheter was secured to the vein with silk sutures and was passed subcutaneously to the top of the skull, where it exited into a connector (a modified 21 gauge cannula; Plastics One) mounted onto the skull with mini-screws and dental cement. Catheters were flushed daily with sterile saline containing gentamycin $(0.08 \mathrm{mg} /$ $\mathrm{ml}$ ) to ensure an open catheter and prevent infection. Rats trained for sucrose self-administration were not implanted with a catheter.

\section{ICES}

Rats were subjected to ICES inside their home cages in the room where the behavioral experiment was conducted under red light luminance. Before stimulation, the rats were allowed a 15 min habituation phase inside the experiment room. They were then connected to the stimulators via an electrical swivel (Plastics One), and stimulation was applied for $30 \mathrm{~min}$ daily for 10 consecutive days. We used two stimulation parameters: $100 \mathrm{~Hz}$, which induces self-stimulation and locomotor activation: [pulse duration, $0.1 \mathrm{~ms}$; intensity, 200-400 $\mu \mathrm{A}$ (Zangen and Shalev,
2003)] and $20 \mathrm{~Hz}$, which is considered safe in TMS treatment in humans (Anderson et al., 2006). For the LH, in the $100 \mathrm{~Hz}$ stimulation, each train consisted of 25 pulses, and two trains per second were used. For the 100 $\mathrm{Hz}$ stimulation in the $\mathrm{PFC}$, each train consisted of 50 pulses, and one train every $2 \mathrm{~s}$ was used, and for the $20 \mathrm{~Hz}$ stimulation, each train consisted of 10 pulses, and one train every $2 \mathrm{~s}$ was used.

\section{Behavioral procedures}

\section{Self-administration}

Apparatus. The self-administration chambers, controlled by a computer system (Med Associates, Georgia, VT), had two levers located $9 \mathrm{~cm}$ above the grid floor, but only one of them (an active, retractable lever) activated the infusion pump. Presses on the inactive, stationary lever were recorded but had no programmed consequences. Each experimental chamber was enclosed in a sound-attenuating wooden box. In the cocaine self-administration experiments, the modified cannula on the rat's skull was connected to a liquid swivel (Med Associates) with polyethylene-50 (PE-50) tubing that was protected by a metal spring. Additional PE-50 tubing connected the swivel to the syringe $(30 \mathrm{ml})$ in the infusion pump that was located outside the chamber. For sucrose self-administration, a $50 \mathrm{ml}$ syringe was attached to the pump and connected to a liquid receptacle located between the two levers.

Training for self-administration. After recovery from surgery, rats were transferred daily into the self-administration chambers. Each daily session, which lasted $3 \mathrm{~h}$ for cocaine-trained and $1 \mathrm{~h}$ for sucrose-trained rats, began with the extraction of the active lever into the chamber and the illumination of a cue light above this lever for $20 \mathrm{~s}$. A house light was turned on for the entire session. A response on the active lever resulted in the delivery of $1 \mathrm{mg} / \mathrm{kg}$ in $0.13 \mathrm{ml}$ (first $3 \mathrm{~d}$ ) or $0.5 \mathrm{mg} / \mathrm{kg}$ (from day 4 until the maintenance criterion was reached) of cocaine and the initiation of a $20 \mathrm{~s}$ timeout period. During this period, lever presses were not reinforced, and the cue light was turned on. Rats trained with sucrose reward were exposed to the same procedure, except that a response on the active lever resulted in the delivery of $0.22 \mathrm{ml}$ sucrose solution into the liquid receptacle. Rats had to reach the conditioning criterion of 4 consecutive days of at least 20 cocaine infusions and 60 sucrose rewards per day before ICES began.

ICES. One day after reaching the conditioning criterion, the rats were randomly divided into two subgroups. The rats in one subgroup underwent ICES treatment as described above ("stimulated" group) and those in the other subgroup served as a sham control ("sham-stimulated" group). Sham-stimulated rats were connected to the electrical cables, but no current was delivered.

Drug-seeking test. The drug-seeking test was conducted $1 \mathrm{~d}$ after the last stimulation session in the self-administration chambers under the same conditions as described above for the training phase, except that cocaine was replaced by saline and sucrose by no reward.

Progressive ratio test. One day after the drug-seeking test, the rats were subjected to a progressive ratio (PR) test, in which increasing numbers of responses are required for successive reinforcements. The test was conducted in the self-administration chambers using the $0.5 \mathrm{mg} / \mathrm{kg} /$ infusion cocaine-dose or $10 \%$ sucrose solution. The initial lever press resulted in delivery of an infusion, but in this test the number of presses required for subsequent infusion was increased sequentially according to the following progression: $1,2,4,6,9,10,15,20,25,32,40,50,62,77,95,118$, $145,178,219$. Concurrent with the start of the infusion, the cue light above the active lever was activated for $20 \mathrm{~s}$ signaling a timeout period. Session was terminated after $90 \mathrm{~min}$ or if rats failed to reach the subsequent infusion for 20 (cocaine) or 30 (sucrose) consecutive minutes. The total number of active lever responses made in the PR session and the number of rewards earned during the PR session were recorded and analyzed.

Evaluation of the electrical dose. Additional groups of rats were used to investigate the effect of ICES of the PFC using a lower (and safer) stimulation frequency on cocaine self-administration. One day after the last self-administration session, the rats were randomly divided into three subgroups. The rats in one subgroup underwent $100 \mathrm{~Hz}$ ICES treatment as described above ("stimulated $100 \mathrm{~Hz}$ " group), and a second subgroup underwent $20 \mathrm{~Hz}$ ICES treatment as described above ("stimulated $20 \mathrm{~Hz}$ " 
group). The third subgroup served as a sham control ("shamstimulated" group). These three groups underwent short-term seeking test and PR test as previously described.

Long-term effects. Nine days after the last ICES treatment of the PFC (using $20 \mathrm{~Hz}, 100 \mathrm{~Hz}$, or sham, as detailed above), rats underwent a seeking test and, on the following day, a PR test to evaluate the long-term effects of the repeated ICES treatment.

\section{Morris water maze test}

Apparatus. The Morris water maze (MWM) consisted of a basin (diameter $130 \mathrm{~cm}$, wall height $40 \mathrm{~cm}$ ) made of gray metal and filled with water $\left(23^{\circ} \mathrm{C}\right)$. The escape platform (diameter $12 \mathrm{~cm}$ ) was made of clear plastic and covered with a grid to ensure a firm grip. The pool was located in a room with various intra- and extra-maze visual cues, such as light fixtures, boxes, and posters.

Procedure. One day after the PR test, rats that had undergone cocaine self-administration were subjected to a MWM test. Each trial began with the rat placed next to the wall and facing the center of the maze at a fixed starting point, with the escape platform hidden $1 \mathrm{~cm}$ beneath water level in the opposite virtual quadrant. Escape latencies were manually recorded. If the rat failed to reach the escape platform within $1 \mathrm{~min}$, it was placed on it for $15 \mathrm{~s}$. Five "acquisition" trials, with $5 \mathrm{~min}$ intertrial intervals, were conducted daily for 4 consecutive days. After each swim, the rat was kept dry in a plastic cage filled with bedding. After the last acquisition trial, a short-term $1 \mathrm{~min}$ probe trial was conducted by removing the platform and placing the rat at the starting point. The time spent in each quadrant was recorded.

General locomotor activity. One day after the probe trial, rats' locomotor activity was measured in a locomotion chamber (see below) for $1 \mathrm{~h}$. There was no habituation phase, and no injections were given.

\section{Behavioral sensitization}

Apparatus. Locomotor activity was monitored in a clear Plexiglas cage $(48 \times 48 \times 48 \mathrm{~cm})$ surrounded by 2 metal frames with $16 \times 16$ infrared light sensors, which allowed recording of horizontal and vertical (rearing) locomotion (Actimot, TSE Systems, Bad Homburg, Germany). The sensor frames were connected to a computer via a control unit. The Actimot software was used for the acquisition and analysis of data. The locomotion chamber and the sensor frames were positioned inside sound-attenuating boxes made of Styrofoam. The experiments were performed in total darkness.

Procedure. Cocaine sensitization treatment: After recovering from surgery, rats were injected intraperitoneally with cocaine $(15 \mathrm{mg} / \mathrm{kg})$ (cocaine-pretreated group) or saline $(0.5 \mathrm{ml}, 0.9 \%)$ (saline-pretreated group) for 7 consecutive days. Before the rats were placed in the locomotion chambers, they were habituated to the experiment room for $15 \mathrm{~min}$ in the dark. Each daily session began with a $30 \mathrm{~min}$ habituation period in the locomotion chamber. Thereafter, the rats were injected with cocaine or saline, and their locomotor activity was recorded for an additional $1 \mathrm{~h}$.

ICES: One day after the last cocaine injection, each experimental group, cocaine-pretreated and saline-pretreated was divided into two subgroups. The rats in one subgroup underwent $100 \mathrm{~Hz}$ ICES treatment as described above ("stimulated" subgroup), and those in the other subgroup served as a sham control ("sham-stimulated" group). Thus, four experimental groups were created for each of the two stimulated brain areas (LH and PFC): cocaine-pretreated stimulated, cocaine-pretreated sham-stimulated, saline-pretreated stimulated, and saline-pretreated sham-stimulated.

Cocaine and saline challenges: One day after ICES treatment, a cocaine challenge $(15 \mathrm{mg} / \mathrm{kg}$, i.p.) was administered to all the rats inside the locomotion chambers under the same conditions as described above. One day after the cocaine challenge, all the rats were subjected to a saline challenge ( $0.5 \mathrm{ml}$, i.p.; $0.9 \%$ ) inside the locomotion chambers under the same conditions.

\section{Histology and immunohistochemistry \\ Histology}

To verify the precise location of the implanted stimulating electrodes and for immunohistochemistry, rats were overdosed with $0.6 \mathrm{ml}$ of pento- barbital $(60 \mathrm{mg} / \mathrm{ml})$. They were then killed and perfused transcardially with PBS and thereafter with 2.5\% paraformaldehyde, $\mathrm{pH} 7.4$, and 5\% sucrose solution. Brains were removed and kept in $1 \%$ paraformaldehyde $/ 30 \%$ sucrose solution until fixation. Brains were frozen and coronal sections $20 \mu \mathrm{m}$ thick (for immunohistochemistry) or $50 \mu \mathrm{m}$ thick (for Nissl staining) were sliced on a microtome and stored after being transferred as free-floating slices to $0.001 \%$ sodium azide solution. To determine where the tips of the electrodes were located, we used a light microscope to inspect the Nissl-stained sections. If the electrode tips were $>0.5$ $\mathrm{mm}$ away from the designated area, the rat was excluded from the analysis (for a detailed diagram, see supplemental Fig. 1, available at www. jneurosci.org as supplemental material).

\section{Immunohistochemistry}

Sections from subregions of the VTA and NAc, obtained from the four experimental groups as described in the locomotion experiments, were used for immunohistochemistry. We examined the levels of NMDA receptor subtype 1 (NR1) using diaminobenzidine tetrahydrochloride (DAB) staining and of glutamate receptor subtype 1 (GluR1) using fluorescence staining. The subregions were anterior VTA (aVTA), posterior VTA (pVTA), anterior NAc (aNAc) core, aNAc shell, posterior NAc (pNAc) core, and pNAc shell. Supplemental Figure 2 (available at www. jneurosci.org as supplemental material) describes the exact location of each subarea. The identification of each subarea was made under fluorescence microscope. The magnification was $10 \times$. For each subarea, a predefined square was constructed and used to set the exact border of the area to be photographed for analysis and quantification (for a detailed diagram, see supplemental Fig. 2, available at www.jneurosci.org as supplemental material). The immunofluorescence slices were divided into groups according to the anatomical subregion. For each VTA slice (anterior and posterior), two pictures were taken: the left and right VTA. For each NAc slice (anterior and posterior), four pictures were taken: the left and right core and the left and right shell. The quantification procedure was as follows. We measured from each picture the overall fluorescence intensity of each predefined square of each subregion using analySIS software (Soft Imaging System, Münster, Germany). The numbers were arbitrary units of intensity.

\section{DAB staining}

Each brain slice was transferred to a plate containing $0.5 \mathrm{ml}$ of PBS in a 24 -well plate. They were then incubated for $30 \mathrm{~min}$ in $200 \mu \mathrm{l}$ of a solution containing $48 \% \mathrm{PBS}, 48 \%$ methanol, $3 \% \mathrm{H}_{2} \mathrm{O}_{2}$, and $1 \% \mathrm{HCl}$ (for inactivation of endogenous peroxidase). They were then incubated in $20 \%$ normal horse serum (NHS) (Vector Laboratories, Burlingame, CA) for $1 \mathrm{~h}$ to block background staining, and this was followed by overnight incubation with mouse anti-NR1 primary antibody (Millipore, Billerica, MA) at a dilution of 1:500 in 2\% NHS at room temperature. The next day, the slices were incubated with biotinylated anti-mouse IgG in $2 \%$ NHS for $1 \mathrm{~h}$, followed by incubation with $\mathrm{A}+\mathrm{B}$ reagents for $1.5 \mathrm{~h}$ at room temperature (Vectastain ABC kit, Vector Laboratories). Finally, peroxidase substrate solution was applied. Developing time, determined online under microscope inspection, was 10-20 min.

\section{Fluorescence staining}

Each brain slice was transferred to a plate containing $0.5 \mathrm{ml}$ of PBS in a 24 -well plate. They were then incubated in $20 \%$ NHS for $1 \mathrm{~h}$ to block background staining, and this was followed by overnight incubation with the rabbit anti-GluR1 primary antibody (Millipore) at dilutions of 1:100 for the NAc and 1:200 for the VTA in 2\% NHS at room temperature. The next day, the slices were incubated with biotinylated anti-rabbit IgG (Vector Laboratories) in 2\% NHS for $1.5 \mathrm{~h}$, followed by incubation with fluorescent-labeled streptavidin (Jackson ImmunoResearch Laboratories, West Grove, PA) at a dilution of 1:200. Developing time, determined on-line under fluorescence microscope inspection, was 20-40 min. To counterstain the nucleus, we applied propidium iodide at a dilution of 1:1000 for $1 \mathrm{~min}$. 
Data analysis

Self-administration

Data from cocaine and sucrose-trained rats were analyzed separately using two-way ANOVA with site (LH vs PFC) and treatment (stimulated vs sham stimulated) as between-subjects factors followed by Fisher's protected least significant difference (PLSD) post hoc analyses to reveal group differences. The dependent measures analyzed in the seeking test were the average rate of infusions earned per hour, and the average rate of responses made on the active and inactive levers per hour, during the test session. In the PR test, the dependent measures analyzed were break points (the number of rewards earned during the PR session, and the total number of active lever responses made during the PR session).

For the electrical "dose" response and the long-term effect experiments, a one-way ANOVA was performed with dose $(100 \mathrm{~Hz}, 20 \mathrm{~Hz}$, sham-stimulated) as the between-subjects factor. The dependent variables were the same as described above.

\section{Locomotor activity}

Activity data were analyzed using three-way ANOVA with site (LH vs PFC), drug (cocaine vs saline), and treatment (stimulated vs shamstimulated) as between-subjects factors, followed by Fisher's PLSD post hoc analyses to reveal group differences. The dependent variable was distance traveled (in meters) in the open field.

\section{Immunohistochemistry}

Data were analyzed using three-way ANOVA, with brain area (aVTA vs pVTA or aNAc core vs aNAc shell vs pNAc core vs pNAc shell), drug (cocaine vs saline), and treatment (stimulated vs sham-stimulated) as between-subjects factors for the four experimental groups (cocainepretreated stimulated, cocaine-pretreated sham-stimulated, salinepretreated stimulated, and saline-pretreated sham-stimulated) followed by Fisher's PLSD post hoc analyses to reveal group differences. This was done separately for the LH and PFC groups because the immunostaining was done on separate days. The dependent variable was an arbitrary unit representing the sum of intensities of DAB or of florescence staining. The saline-pretreated sham stimulation groups were considered as baseline levels, and all the groups were calculated as the change in percentage from the average measure obtained in that group. All data are presented as means \pm SEM of the percentage, and significant differences are reported for $p<0.05$

\section{Results}

\section{Self-administration}

Rats learned quickly to press the lever for intravenous cocaine, and after 9-13 d reached a maintenance criterion in which at least 20 infusions per session were obtained over 4 consecutive days. Rats pressing for sucrose reached after $6-8 \mathrm{~d}$ a maintenance criterion in which at least sixty sucrose rewards per session were obtained over 4 consecutive days. The mean numbers of infusions (cocaine) or rewards (sucrose) taken by rats from comparable groups (see Materials and Methods) were similar during the acquisition phase until maintenance criterion was reached. Selfadministration was followed by real or sham ICES sessions of the medial forebrain bundle, at the level of the LH or the PFC for 10 consecutive days. Thereafter, behavioral tests were performed.

\section{Effects of $100 \mathrm{~Hz}$ ICES treatment of the LH or the PFC on cocaine-seeking behavior}

One day after the last $100 \mathrm{~Hz}$ ICES session, rats were subjected to a reward-seeking test in which cocaine was replaced by saline. A two-way ANOVA revealed a significant treatment effect $\left(F_{(1,36)}=\right.$ $10.2, p<0.003$ ) indicating that $100 \mathrm{~Hz}$ ICES of both the LH ( post $h o c, p<0.04)$ (Fig. $1 A)$ or the PFC $(p<0.05)$ (Fig. $1 A)$ significantly reduced cocaine-seeking behavior relative to that of shamstimulated rats, as measured by the average rate of active lever responses per hour. The effect of $100 \mathrm{~Hz}$ ICES on cocaine-seeking
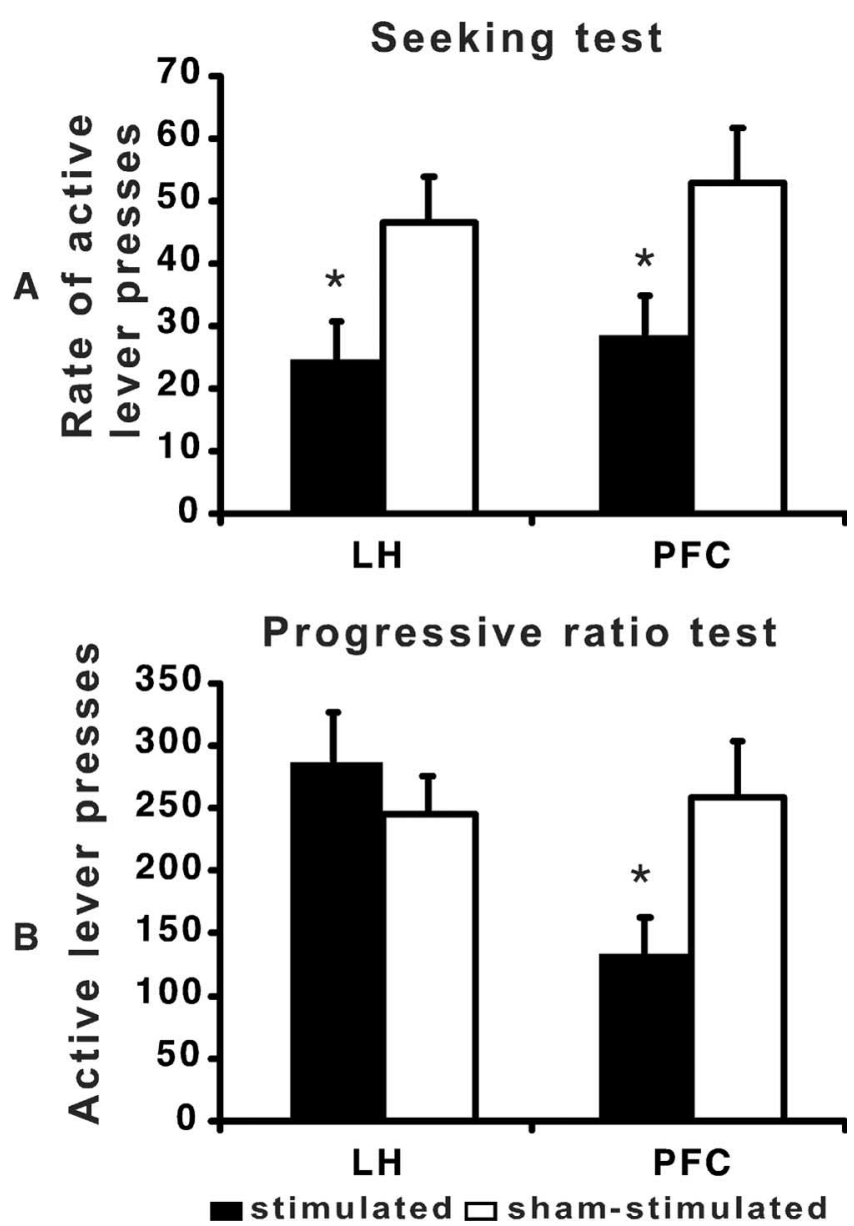

Figure 1. Effects of ICES treatment of the LH or the PFC on cocaine self-administration. Each administration provided $0.5 \mathrm{mg} / \mathrm{kg}$ cocaine intravenously and was followed by a 20 s time-out in which active lever responses were counted but were not reinforced. After reaching the maintenance criterion for cocaine infusion (LH: stimulated, $n=14$; sham-stimulated, $n=9$; PFC: stimulated, $n=8$; sham-stimulated, $n=9$ ), rats were subjected to ICES treatment in their home cages for $10 \mathrm{~d}$. $\boldsymbol{A}$, Average rate of active lever presses per hour performed during the reward-free seeking test conducted $1 \mathrm{~d}$ after ICES treatment. $\boldsymbol{B}$, Numbers of active lever presses during the 90 min progressive ratio test conducted $1 \mathrm{~d}$ after the seeking test. Data are mean values \pm SEM. ${ }^{*} p<0.05$ using two-way ANOVA followed by Fisher's PLSD post hoc tests.

behavior did not differ between LH and PFC rats as the site by treatment interaction was not significant $\left(F_{(3,17)}=0.5, p=0.47\right)$. There were no ICES-induced significant effects on the nonactive lever responses during the cocaine-seeking test in either the LH or PFC rats $(p>0.05)$.

\section{Effects of $100 \mathrm{~Hz}$ ICES treatment of the LH or the PFC on cocaine self-administration under a progressive ratio schedule}

One day after the drug-seeking test, the rats underwent a PR test, in which they needed to progressively increase the number of active lever responses to receive a subsequent reward. We compared the total numbers of active lever responses made during the PR session by rats subjected to $100 \mathrm{~Hz}$ ICES of the LH or the PFC and by the corresponding sham-stimulated subgroups. A twoway ANOVA revealed a significant site by treatment interaction $\left(F_{(1,28)}=4.97, p<0.04\right)$. Further analysis revealed that rats treated with $100 \mathrm{~Hz}$ ICES of the PFC (post hoc, $p<0.05$ ) (Fig. $1 B)$, but not of the LH $(p>0.05)$ (Fig. $1 B)$, demonstrated significantly fewer active lever responses than sham-stimulated rats. This was also reflected in the number of rewards earned by the 

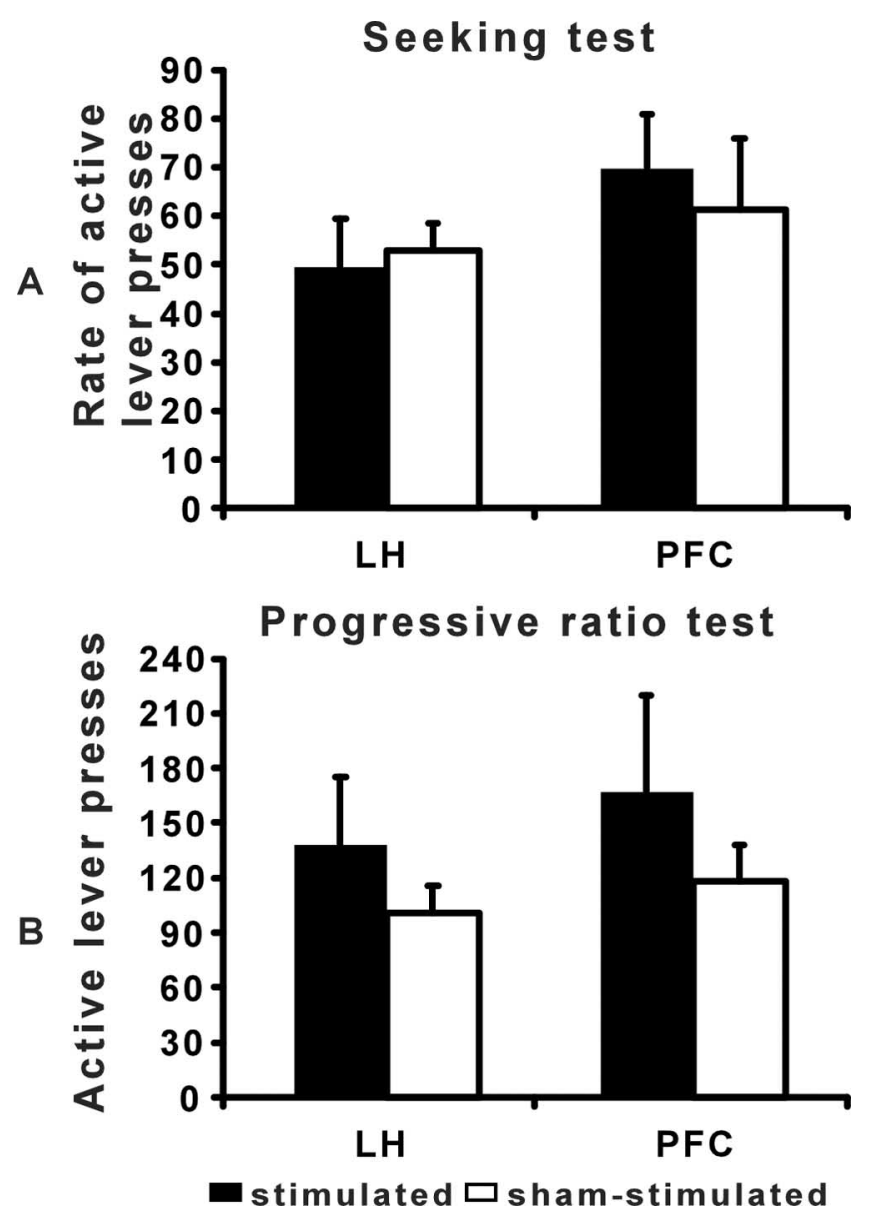

Figure 2. Effects of ICES treatment of the LH or the PFC on sucrose self-administration. Each administration provided $10 \%$ sucrose solution into a liquid receptacle and was followed by a $20 \mathrm{~s}$ time-out in which active lever responses were counted but were not reinforced. After reaching the maintenance criterion for sucrose consumption (LH and PFC: stimulated, $n=7$; sham-stimulated, $n=7$ ), rats were subjected to ICES treatment as in Figure 1. $\boldsymbol{A}$, Rate of active lever presses during the reward-free seeking test $(60 \mathrm{~min}$ ) conducted $1 \mathrm{~d}$ after ICES treatment. $\boldsymbol{B}$, Numbers of active lever presses during the 90 min progressive ratio test conducted $1 \mathrm{~d}$ after the seeking test. Data are mean values \pm SEM.

rats during the PR session, the "break point," which was significantly lower in PFC-ICES-treated (but not LH-ICES-treated) rats, compared with the sham-stimulated ones (PFC, ICES $10.1 \pm 0.8$ vs sham-stimulated, $12.7 \pm 0.6 ; t=2.44, p<0.03)$. There were no ICES-induced significant effects on the nonactive lever responses during the PR test in either the LH or PFC rats $(p>0.05)$.

\section{Effects of $100 \mathrm{~Hz}$ ICES treatment of the LH or the PFC on} sucrose-seeking behavior and on sucrose self-administration under a progressive ratio schedule

We conducted the same behavioral experiments on sucrosetrained rats to examine the effect of $100 \mathrm{~Hz}$ ICES on a natural reward. A two-way ANOVA did not reveal any significant effects in the sucrose-seeking test (Fig. 2A) or in the PR test (Fig. $2 B$ ), indicating that $100 \mathrm{~Hz}$ ICES of either the LH or the PFC did not affect the rate of active lever responses. Therefore, $100 \mathrm{~Hz}$ ICES treatment of either the PFC or the LH reduced reward-seeking behavior for cocaine but not for a natural reward. In addition, $100 \mathrm{~Hz}$ ICES treatment of the PFC, but not of the LH, decreased motivation for cocaine intake, whereas response rate obtained with a natural reward was not affected by $100 \mathrm{~Hz}$ ICES treatment of either of the stimulation sites.

Effects of ICES treatment on performance in the Morris water maze and on locomotor activity

One day after the PR test, we examined whether the observed effects of ICES treatment in the cocaine-trained rats were attributable to a general disruption of learning and memory or to nonspecific motor deficits. The effects of the ICES treatment on learning and memory were assessed using the MWM paradigm, and potential motor deficits were assessed by monitoring spontaneous locomotor activity.

ICES did not disrupt the ability to learn the MWM task. Repeated-measures ANOVA performed on the $4 \mathrm{~d}$ acquisition phase data in both the LH-treated and the PFC-treated rats revealed a significant time effect for both treatment areas [ $\mathrm{LH}-$ treated rats, $F_{(3,13)}=75.5, p<0.0001$ (Fig. 3A); PFC-treated rats, $F_{(3,17)}=71.8, p<0.0001$ (Fig. $3 B$ )], but no group or group by time interaction effects [LH-treated rats, $F_{(1,3)}=0.5, p=0.49$ (Fig. 3A); PFC-treated rats, $F_{(1,3)}=0.6, p=0.8$ (Fig. $3 B$ )]. Similarly, in the probe trial both the stimulated and the shamstimulated rats from the LH-treated groups (ANOVA, $F_{(3,14)}=$ $12, p<0.0001$ ) (Fig. 3C) and the PFC-treated groups $\left(F_{(3,18)}=\right.$ $20, p<0.0001$ ) (Fig. 3D) spent significantly more time in the quadrant where the hidden ramp was previously stationed (Q1) than in the other three quadrants, and stimulation of either site did not affect the percentage of time spent in Q1 (Mann-Whitney test, $p=0.99$ and $p=0.54$ for the LH-treated and PFC-treated groups, respectively).

After the MWM probe test, spontaneous locomotor activity was monitored for $1 \mathrm{~h}$ in chambers equipped with photobeam sensors that detect both horizontal and vertical (rearing) activity. No significant differences were found between the stimulated and sham-stimulated subgroups in distance traveled or number of rearing movements, for LH-treated and PFC-treated rats (data not shown).

\section{Induction of psychomotor sensitization}

The effects of ICES on the expression of psychomotor sensitization were tested in a different cohort of rats. A gradual increase in the cocaine's psychomotor activating effects was observed over the seven daily injections, indicating the induction of behavioral sensitization in the cocaine-pretreated rats relative to salinepretreated rats (data not shown). Repeated-measures ANOVA revealed significant cocaine-treatment $\left(\mathrm{LH}, F_{(1,15)}=228, p<\right.$ 0.0001 ; PFC, $\left.F_{(1,15)}=73, p<0.0001\right)$ and time $\left(\mathrm{LH}, F_{(1,6)}=2.23\right.$, $p<0.05$; PFC, $\left.F_{(1,6)}=3, p<0.01\right)$ effects in the LH and PFC groups.

\section{Effects of ICES treatment of the LH or the PFC on the} expression of psychomotor sensitization

After the induction of behavioral sensitization, $10 \mathrm{~d}$ of $100 \mathrm{~Hz}$ ICES treatment of the LH or PFC was conducted in the same manner as described above for the self-administration paradigm. After rats had completed the $10 \mathrm{~d}$ ICES or sham stimulation treatment, we examined the expression of behavioral sensitization by administering a cocaine challenge to all LH-treated (Fig. $4 A$ ) and all PFC-treated (Fig. $4 B$ ) rats. Three-way ANOVA revealed significant drug $\left(F_{(1,51)}=36.9, p<0.0001\right)$ and site $\left(F_{(1,51)}\right.$ $=4.0, p<0.05)$ main effects and site by treatment interaction $\left(F_{(1,51)}=8.4, p<0.01\right)$. Separate analysis in the sham-stimulated groups revealed that the distance traveled by the cocainepretreated sham-stimulated rats [LH, $n=6$ (Fig. $4 E$ ); PFC, $n=7$ 
(Fig. $4 F)]$ was significantly higher $\left(F_{(1,23)}\right.$ $=16.6, p<0.001)$ than that traveled by saline-pretreated sham-stimulated ones (LH, $n=7$; PFC, $n=7$ ), indicating expression of psychomotor sensitization. Additionally, post hoc analysis revealed that in the LH-stimulated cocaine-pretreated rats $(n=8)$, the postinjection distance traveled was significantly higher $(p<0.05)$ than that of cocaine-pretreated rats receiving sham stimulation (Fig. 4E). No significant effect of ICES treatment was detectable in the saline-pretreated rats, indicating the specificity of the ICES effect for the cocaine-pretreated rats only. On the other hand, when ICES was applied to the PFC, it significantly decreased the postinjection distance traveled for cocaine-pretreated rats $(n=8)$, compared with cocaine shamstimulated rats $(p<0.05)$ (Fig. $4 F)$. Thus, ICES treatment of the PFC decreased the expression of cocaine psychomotor sensitization, whereas ICES treatment of the LH induced an opposite effect.

It is also important to note that although ICES of the PFC had no significant effect on locomotion of the salinepretreated rats during the postinjection phase, it increased locomotion in these rats during the habituation phase $(p<0.05)$ (Fig. 4D), indicating that the decreased psychomotor response to cocaine observed in rats receiving ICES treatment of the PFC (Fig. $4 F$ ) could not be attributed to a general reduction in activity.

\section{Effects of ICES treatment of the LH or the PFC on locomotor activity after a saline challenge}

One day after the cocaine-challenge test, conditioned locomotion was assessed in the LH- and PFC-stimulated groups after a saline challenge (Fig. 5). Three-way ANOVA revealed a significant drug main effect $\left(F_{(1,51)}=14.5, p<0.001\right)$ and drug by treatment interaction $\left(F_{(1,51)}=5.3, p<0.03\right)$. Separate analysis in the sham-stimulated groups revealed that the distance traveled by the cocaine-pretreated sham-stimulated rats $[\mathrm{LH}$ (Fig. 5C); PFC (Fig. $5 D)]$ was significantly higher $\left(F_{(1,25)}=17.5, p<0.001\right)$ than that traveled by saline-pretreated sham-stimulated ones, indicating expression of conditioned locomotion. Importantly, post hoc analysis revealed that LH-ICES treatment of cocaine-pretreated rats significantly decreased distance traveled compared with the sham controls $(p<0.02)$ (Fig. $5 C)$. Moreover, among the salinepretreated rats there were no significant differences in locomotion between the LH-ICES-treated animals and the sham controls, indicating specificity of the ICES effect for the cocainepretreated rats (in which conditioned locomotion was observed). Furthermore, locomotion during the $30 \mathrm{~min}$ of the habituation phase did not differ significantly between LH-ICES-treated and sham controls $(p>0.05)$ (Fig. $5 A$ ), indicating that the observed postinjection decrease in locomotion did not result from a general reduction in activity caused by the ICES treatment. On the other hand, PFC-stimulated rats showed no significant ICESinduced differences in locomotion during either the habituation (Fig. $5 B$ ) or the postinjection phases (Fig. 5D).
LH Rats
PFC Rats

Acquisition Phase

Figure 3. Effects of ICES treatment of the LH or PFC on performance in the Morris water maze. Latency to the hidden platform during $4 \mathrm{~d}$ of training. On each training day rats were allowed five $60 \mathrm{~s} \mathrm{trials.} \mathrm{Data} \mathrm{are} \mathrm{the} \mathrm{daily} \mathrm{mean} \mathrm{latency} \pm$ SEM over the five , $\boldsymbol{B}$, Repeated-measures ANOVA revealed a significant time effect for both treatment areas $\mathrm{LH}$-treated rats $(\boldsymbol{A})$ and

Probe Test

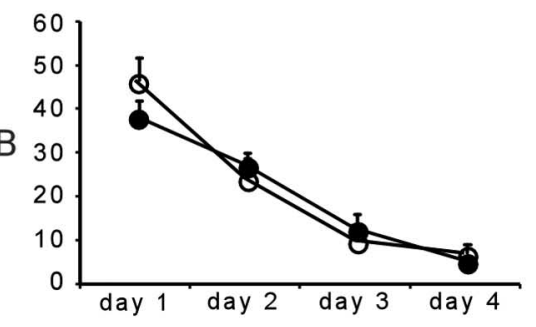

Stimulated

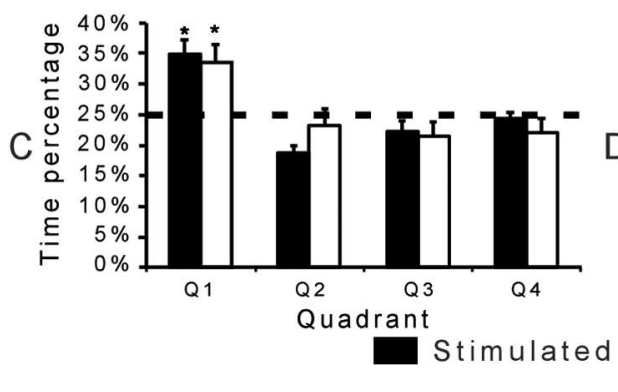

Sham-Stin

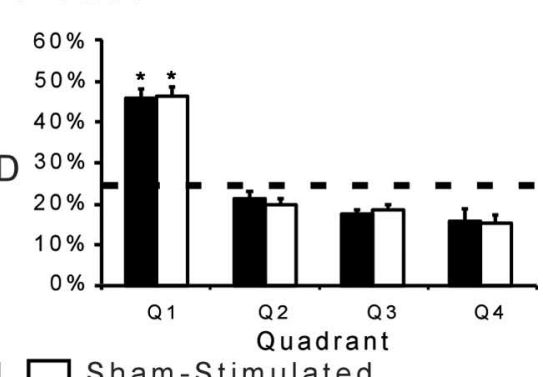
to

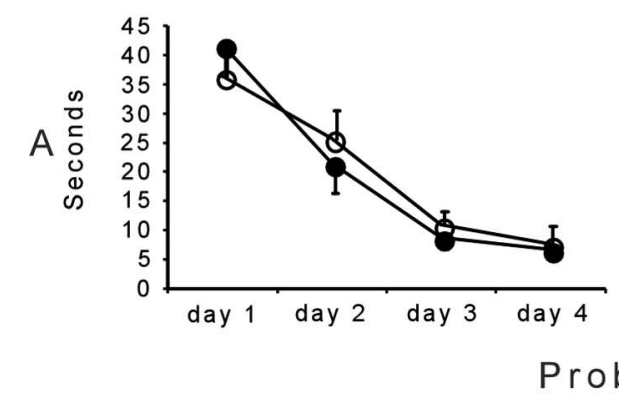

ICES treatment of the LH or PFC did not affect the time spent in each quadrant during the probe test. ${ }^{*} p<0.001$ (Q1 compared

The effect of stimulation frequency on short- and long-term alterations in cocaine-seeking behavior and cocaine selfadministration under a progressive ratio schedule Repeated ICES of the PFC using $100 \mathrm{~Hz}$ reduced the expression of cocaine-induced psychomotor sensitization as well as cocaineseeking and cocaine self-administration under a progressive ratio schedule, suggesting the potential application of such treatment approach in addiction. Therefore, additional groups of rats were used to compare the effect of $100 \mathrm{~Hz}$ ICES to $20 \mathrm{~Hz}$ ICES ("dose effect") and to test whether attenuation of drug seeking and selfadministration in a PR test would also be evident 9-10 d ("long term") and not just 1-2 d ("short term") after the $10 \mathrm{~d}$ of ICES treatment.

\section{Short-term (1-2d) effect of the ICES treatment}

One-way ANOVA of the rate of active lever responses in the cocaine-seeking test performed $1 \mathrm{~d}$ after termination of ICES treatment revealed a significant effect of dose (frequency) $\left(F_{(2,26)}\right.$ $=5.7, p<0.01)$. Post hoc analysis revealed that the rate of active lever responses was significantly lower in rats receiving either the $100 \mathrm{~Hz}$ ICES $(p<0.005)$ or the $20 \mathrm{~Hz}$ ICES $(p<0.04)$ compared with sham-stimulated rats (Fig. 6A). There were no ICESinduced significant effects on the nonactive lever responses during the seeking test $(p>0.05)$.

Comparing the total number of active lever responses made during the PR session using a one-way ANOVA revealed a significant dose effect $\left(F_{(2,26)}=5.3, p<0.02\right)$. Post hoc analysis revealed that the rate of active lever responses was significantly lower in rats receiving $100 \mathrm{~Hz}$ ICES $(p<0.005)$ or in rats receiving $20 \mathrm{~Hz}$ ICES $(p<0.03)$ compared with sham-stimulated rats (Fig. 6C). This indicates that both $100 \mathrm{~Hz}$ and $20 \mathrm{~Hz}$ ICES treatment of the PFC decreased motivation for cocaine intake. 


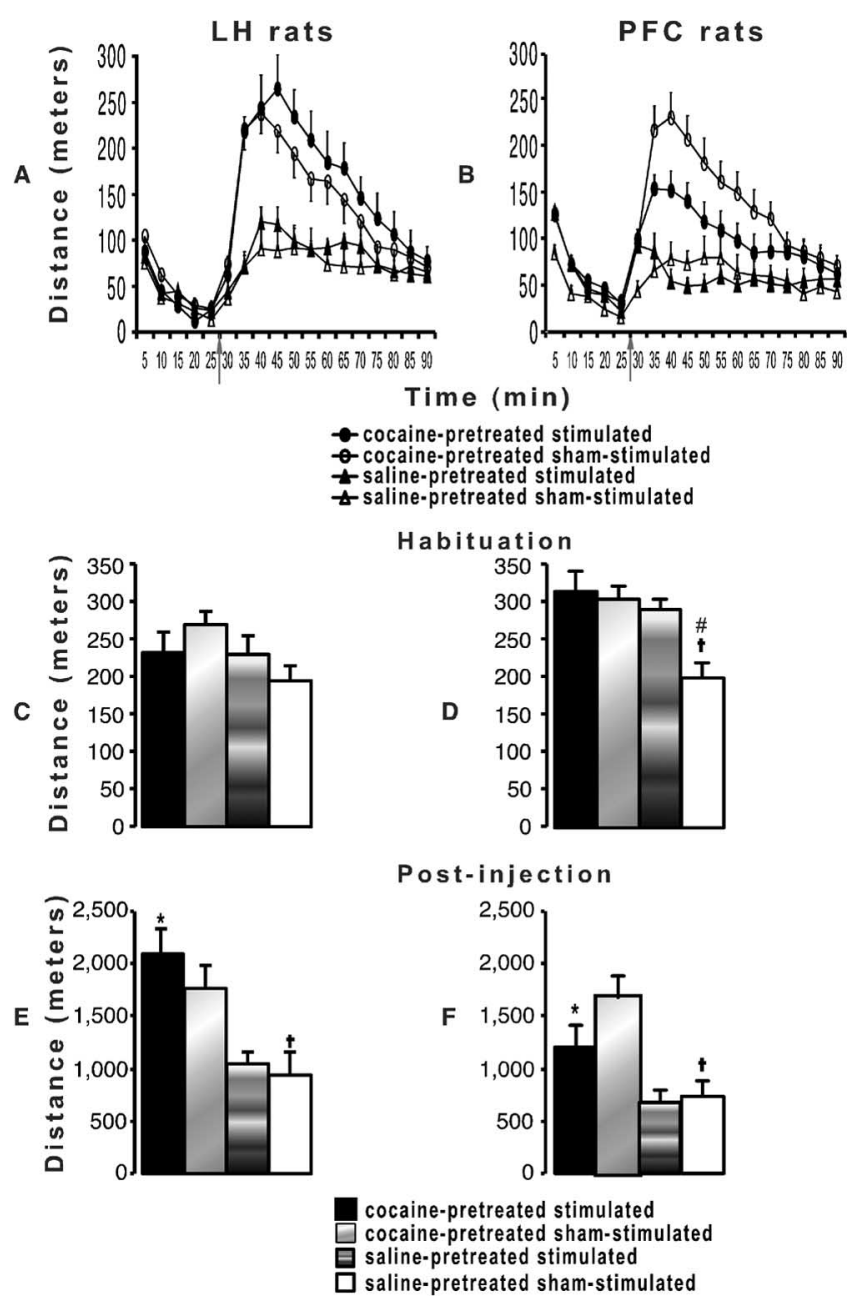

Figure 4. Effects of ICES treatment of the LH or PFC on locomotion in an open field during a cocaine challenge. $A, B$, Distance traveled in response to cocaine challenge $(15 \mathrm{mg} / \mathrm{kg}$, i.p.) comprising a habituation phase $(30 \mathrm{~min})$ and a postinjection phase $(60 \mathrm{~min})$. Arrow indicates time of injection. $\boldsymbol{C}, \boldsymbol{D}$, Cumulative distance during habituation phase. $\boldsymbol{E}, \boldsymbol{F}$, Cumulative distance during postinjection phase. Data are mean distances traveled \pm SEM and analyzed using threeway ANOVA followed by Fisher's PLSD post hoc tests. ${ }^{*} p<0.05$ (cocaine-pretreated stimulated vs cocaine-pretreated sham-stimulated). ${ }^{\dagger} p<0.05$ (cocaine-pretreated sham-stimulated vs saline-pretreated sham-stimulated). ${ }^{\#} p<0.05$ (saline-pretreated stimulated vs salinepretreated sham-stimulated).

Long-term (9-10 d) effect of the ICES treatment

Similar to the effects observed in the short-term measurements, one-way ANOVA on the rate of active lever responses in the cocaine-seeking test revealed a significant dose (frequency) effect $\left(F_{(2,18)}=3.6, p<0.05\right)$. Post hoc analysis revealed that the rate of active lever responses was significantly lower in rats receiving 100 $\mathrm{Hz}$ ICES $(p<0.04)$ or in rats receiving $20 \mathrm{~Hz}$ ICES $(p<0.03)$ compared with sham-stimulated rats (Fig. $6 B$ ). This indicates that the effect of $100 \mathrm{~Hz}$ and $20 \mathrm{~Hz}$ ICES on cocaine-seeking behavior can last for at least $9 \mathrm{~d}$ after the ICES treatment.

Comparing the total number of active lever responses made during the PR session using a one-way ANOVA revealed a significant dose effect $\left(F_{(2,18)}=3.6, p<0.05\right)$. However, further post hoc analysis revealed that only rats receiving $20 \mathrm{~Hz}$ ICES $(p<$ 0.02 ) but not rats receiving $100 \mathrm{~Hz}$ ICES ( $p=0.066)$ demonstrated significantly fewer active lever responses than shamstimulated rats (Fig. 6D).
LH rats PFC rats

Habituation

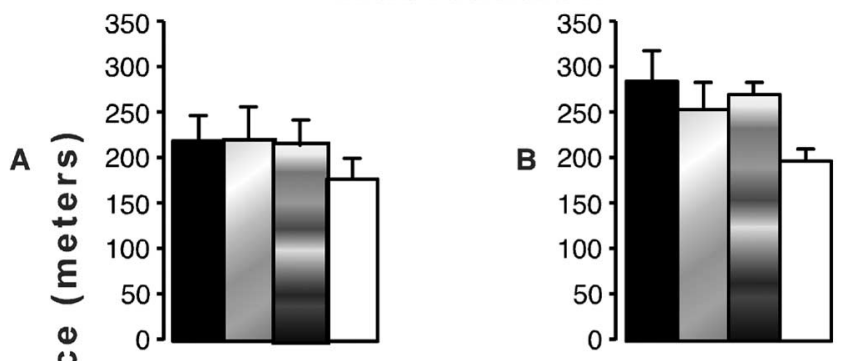

\section{Post-injection}
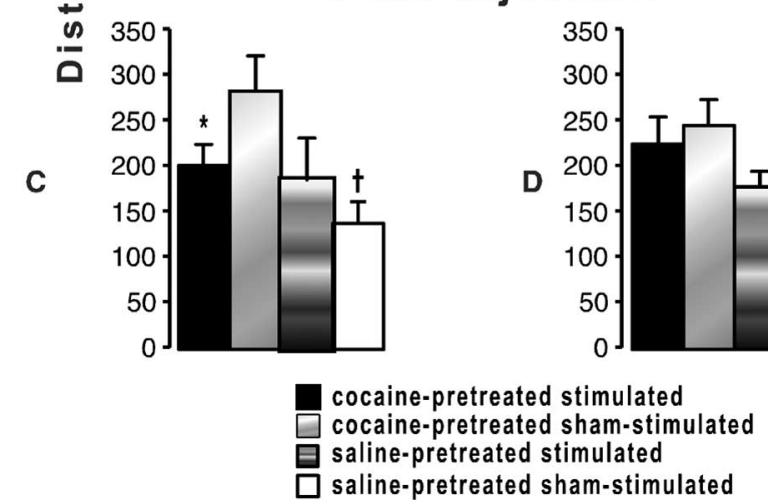

Figure 5. Effects of ICES treatment of the LH or PFC on locomotion in an open field during a saline challenge. Distance traveled in response to a saline challenge comprising a habituation phase (30 $\mathrm{min}$ ) and a postinjection phase $(60 \mathrm{~min}) . \boldsymbol{A}, \boldsymbol{B}$, Cumulative distance during habituation phase. $\boldsymbol{C}, \mathbf{D}$, Cumulative distance during postinjection phase. Data are mean distances traveled \pm SEM analyzed using three-way ANOVA followed by Fisher's PLSD post hoc tests. ${ }^{*} p<0.05$ (cocaine-pretreated stimulated vs cocaine-pretreated sham-stimulated). ${ }^{\dagger} p<0.05$ (cocaine-pretreated sham-stimulated vs saline-pretreated sham-stimulated).

\section{Effects of ICES treatment of the LH or the PFC on AMPA} glutamate receptor subtype 1 (GluR1) and on NMDA receptor subtype 1 (NR1) in the VTA and NAc

Immediately after the end of the behavioral sensitization experiments, rats were killed and brains were taken for analysis of the levels of GluR1 and NR1 in subregions of the VTA and NAc using immunofluorescence. Tables 1 and 2 present the effects of the different treatment conditions on glutamate receptor subtype levels as percentage change from the saline-pretreated shamstimulated groups (serving as the $100 \%$ baseline), in the specified subregions. See Materials and Methods and the supplemental material (available at www.jneurosci.org) for a full description of the subdivision technique and quantification of the immunofluorescence. We used immunofluorescence rather than immunoblotting because of the high anatomical precision that could be achieved. Receptor subtype levels, recorded as arbitrary intensity units, were analyzed using three-way ANOVA followed by Fisher's PLSD post hoc tests where appropriate, and significant group differences are specified in the tables. We describe in the text only results that show that ICES induced the opposite change in receptor levels from repeated cocaine.

\section{Effects of LH-ICES and PFC-ICES treatment on GluR1} expression in the VTA and NAc

For the LH-stimulated rats, a three-way ANOVA of GluR1 levels in the aVTA and pVTA revealed significant drug $\left(F_{(1,53)}=14.6\right.$, $p<0.001)$ and treatment $\left(F_{(1,53)}=15.1, p<0.001\right)$ main effects and significant drug by treatment $\left(F_{(1,53)}=15.7, p<0.001\right)$ and 


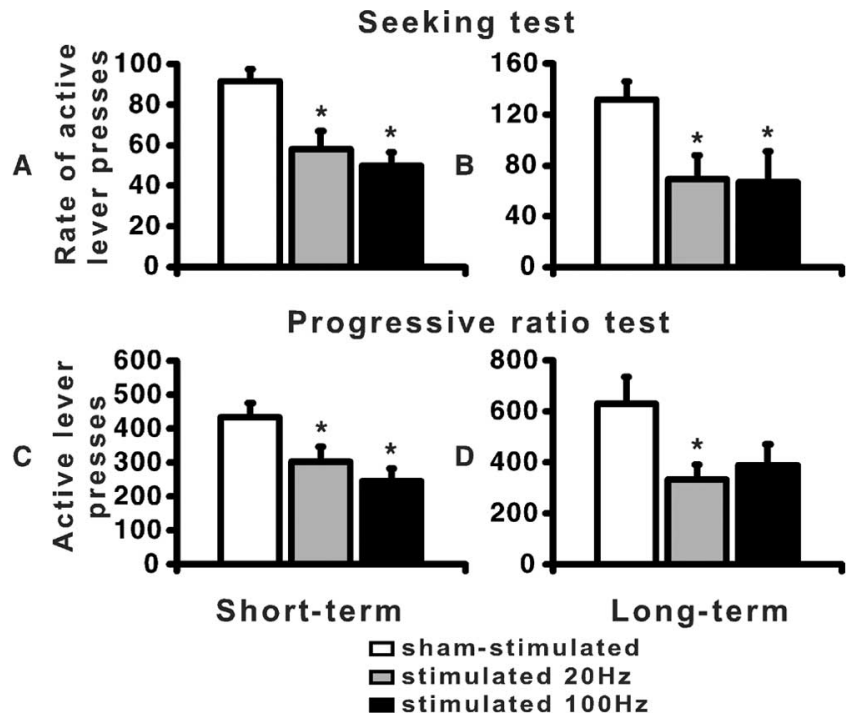

Figure 6. Effects of different ICES frequencies (dose-response) of the PFC on cocaine selfadministration. Each administration provided $0.5 \mathrm{mg} / \mathrm{kg}$ cocaine intravenously, and was followed by a 20 s time-out in which active lever responses were counted but were not reinforced. After reaching the maintenance criterion for cocaine infusion, rats were subjected to $100 \mathrm{~Hz}$ or $20 \mathrm{~Hz}$ ICES treatment in their home cages for $10 \mathrm{~d}$ (stimulated $100 \mathrm{~Hz}, n=8$; stimulated $20 \mathrm{~Hz}$, $n=6$; sham-stimulated, $n=9$ ). $A$, Average rate of active lever presses per hour performed during the reward-free short-term seeking test conducted $1 \mathrm{~d}$ after completion of the $10 \mathrm{~d}$ ICES treatment. $\boldsymbol{B}$, Average rate of active lever presses per hour performed during the reward-free long-term seeking test conducted $9 \mathrm{~d}$ after the completion of the $10 \mathrm{~d}$ ICES treatment. $\boldsymbol{C}$ Numbers of active lever presses during the 90 min short-term progressive ratio test conducted $1 \mathrm{~d}$ after the short-term seeking test. $\boldsymbol{D}$, Numbers of active lever presses during the $90 \mathrm{~min}$ long-term progressive ratio test conducted $1 \mathrm{~d}$ after the long-term seeking test. Data are mean values \pm SEM. ${ }^{*} p<0.05$ using one-way ANOVA followed by Fisher's PLSD post hoc tests.

drug by treatment by brain area $\left(F_{(1,53)}=15.2, p<0.001\right)$ interactions. This indicates that repeated cocaine administration upregulated GluR1 levels in the aVTA and pVTA. Thereafter, a separate analysis revealed that LH-ICES treatment reversed the cocaine-induced upregulation of GluR1 expression in the aVTA $\left(F_{(1,26)}=16.1, p<0.001\right.$, see specific post hoc tests in Table 1$)$ but not in the pVTA $\left(F_{(1,27)}=0.1, p>0.05\right)$, and had no effect on saline-pretreated rats (Table 1 and Fig. 7). No additional significant effects of LH-ICES treatment on the GluR1 levels in the VTA or NAc were found.

For the PFC-stimulated rats, a three-way ANOVA of GluR1 levels in the anterior (aNAc) and posterior ( $\mathrm{pNAc}$ ) core and shell revealed a significant brain area $\left(F_{(3,102)}=5.85, p<0.002\right)$ main effect and significant drug by treatment $\left(F_{(1,102)}=38.11, p<\right.$ $0.0001)$ and drug by brain area by treatment $\left(F_{(3,102)}=4.1, p<\right.$ $0.01)$ interactions. Thereafter, a separate analysis revealed a significant drug effect $\left(F_{(1,56)}=22.1, p<0.0001\right)$ in the shamstimulated groups, indicating that repeated cocaine downregulated GluR1 levels in the subregions of the NAc. Importantly, in the aNAc shell and core but not pNAc shell and core, PFC-ICES treatment reversed the cocaine-induced downregulation of GluR1 $\left(F_{(3,46)}=7.5, p<0.001\right.$, see specific post hoc comparisons in Table 1). No additional significant effects of PFC-ICES treatment on the GluR1 levels in the VTA or NAc were found (Table 1).

\section{Effects of LH-ICES and PFC-ICES treatment on NR1 expression in the VTA and NAc}

In the LH-stimulated rats, ICES did not induce significant differences in NR1 expression, in any of the subregions of the VTA and NAc (Table 2).
For the PFC-stimulated rats, a three-way ANOVA of NR1 levels in the aNAc and pNAc revealed a significant drug by treatment interaction $\left(F_{(1,52)}=6.0, p<0.02\right)$. Thereafter, a separate analysis revealed a significant drug effect $\left(F_{(1,28)}=4.2, p<0.05\right)$ in the sham-stimulated groups indicating that repeated cocaine downregulated NR1 levels in the aNAc and pNAc. In addition, a significant treatment effect $\left(F_{(1,28)}=6.9, p<0.02\right)$ in the cocaine pretreated groups was found, indicating that PFC-ICES increased NR1 levels in the aNAc and pNAc and reversed the downregulation caused by repeated cocaine (see specific post hoc comparisons in Table 2).

\section{Discussion}

Cocaine addiction-related behaviors were shown in this study to be affected by repeated ICES treatment, whereas sucrose-taking behavior remained unchanged. The ICES treatment effects were site specific. ICES of the PFC reversed the psychomotor sensitization induced by repeated exposure to cocaine and attenuated cocaine self-administration (in the PR tests) as well as cocaineseeking behavior (under extinction conditions). In contrast, ICES of the LH sensitized the psychomotor sensitization induced by repeated cocaine exposure but attenuated cocaineconditioned locomotor response, and reduced cocaine-seeking behavior only under extinction conditions (but not when the drug was available in the PR tests). The ICES treatment effects were specific to cocaine-trained rats, as the same treatment did not affect sucrose-trained rats, ruling out the possibility of a generalized effect on motor performance or reward-motivated behavior.

Repeated exposure to drugs of abuse results in neuronal adaptations that might be critical in the development and maintenance of drug addiction (Kalivas and Volkow, 2005). Repeated electrical brain stimulation, which is hypothesized to cause activation of brain circuits that are usually activated by natural rewards and drugs of abuse (Wise, 1996), also results in neuronal adaptations in reward-related areas (Arvanitogiannis et al., 2000).

Previous studies evaluating the interaction between electrical stimulation and repeated drug use found lack of sensitization to the facilitating effects of cocaine on intracranial self-stimulation (Bauco and Wise, 1997), and that repeated LH self-stimulation delayed the development of behavioral sensitization to repeated administration of amphetamine (Wise and Munn, 1993). On the other hand, electrical stimulation of the VTA sensitized rats to the acute psychomotor activating effects of amphetamine (BenShahar and Ettenberg, 1994).

Although intracranial stimulation of the LH and PFC activate some overlapping reward-related brain areas (Arvanitogiannis et al., 2000), the different effects of ICES treatment in these two regions can be explained by their different circuitries (Yadin et al., 1983), electrophysiologic characteristics (Schenk and Shizgal, 1982), and drug sensitivity (Hand and Franklin, 1983). Based on these studies and our present findings, we suggest that repeated LH-ICES treatment strongly hyperactivates the reward system, causing hypersensitization in circuits mediating the response to subsequent cocaine challenge. In contrast, this same treatment, through a mechanism discussed below, attenuates the conditioned response to cocaine-associated cues, as observed in both the locomotor response to a saline challenge and the drug-free seeking test. PFC-ICES treatment, presumably by activating different reward-related circuits, reduced the sensitivity to cocaine, as demonstrated by lower self-administration rate and breaking point in the PR test and attenuated psychomotor response to 
Table 1. Relative levels of the GluR1 receptor subtype in subregions of the ventral tegmental area and nucleus accumbens of rats subjected to ICES treatment of the lateral hypothalamus or the prefrontal cortex

\begin{tabular}{lcccc}
\hline & $\begin{array}{l}\text { Cocaine- } \\
\text { stimulated }\end{array}$ & $\begin{array}{l}\text { Cocaine } \\
\text { sham-stimulated }\end{array}$ & $\begin{array}{l}\text { Saline- } \\
\text { stimulated }\end{array}$ & $\begin{array}{l}\text { Saline } \\
\text { sham-stimulated }\end{array}$ \\
\hline $\begin{array}{l}\text { GluR1 in LH-treated rats } \\
\text { aNAc core }\end{array}$ & $61 \pm 22$ & $17 \pm 4^{b}$ & $81 \pm 40$ & $100 \pm 24$ \\
aNAc shell & $56 \pm 21$ & $39 \pm 14^{b}$ & $107 \pm 48$ & $100 \pm 20$ \\
pNAc core & $50 \pm 13$ & $16 \pm 3$ & $52 \pm 24$ & $100 \pm 42$ \\
pNAc shell & $37 \pm 10$ & $23 \pm 4^{b}$ & $41 \pm 20$ & $100 \pm 37$ \\
aVTA & $54 \pm 38^{a}$ & $763 \pm 213^{b}$ & $119 \pm 43$ & $100 \pm 23$ \\
pVTA & $140 \pm 25$ & $158 \pm 38^{b}$ & $88 \pm 15$ & $100 \pm 20$ \\
GluR1 in PFC-treated rats & $123 \pm 40^{a}$ & $36 \pm 14^{b}$ & $70 \pm 15$ & $100 \pm 36$ \\
aNAc core & $267 \pm 107^{a}$ & $16 \pm 4^{b}$ & $35 \pm 10$ & $100 \pm 38$ \\
aNAc shell & $36 \pm 16$ & $16 \pm 3^{b}$ & $18 \pm 5^{c}$ & $100 \pm 25$ \\
pNAc core & $29 \pm 13$ & $19 \pm 5^{b}$ & $18 \pm 3^{c}$ & $100 \pm 27$ \\
pNAc shell & $394 \pm 90$ & $395 \pm 83^{b}$ & $77 \pm 11$ & $100 \pm 19$ \\
aVTA & $127 \pm 23$ & $140 \pm 21$ & $129 \pm 13$ & $100 \pm 20$ \\
pVTA & & & & \\
\hline
\end{tabular}

Rats were perfused immediately after the saline challenge, and slices were immunolabeled for GluR1. Data are presented as percentage (means \pm SEM) of the average fluorescence intensity measured in the saline-pretreated sham-stimulation groups that were considered as baseline levels for each subregion. Significant differences are reported for $p<0.05$. ${ }^{a}$ Significantly different from cocaine-pretreated sham-stimulated; ${ }^{b}$ Significantly different from salinepretreated sham-stimulated; 'Significantly different from saline-pretreated sham-stimulated.

Table 2. Immunolabeling of the NR1 receptor subtype in subregions of the ventral tegmental area and nucleus accumbens of rats subjected to ICES of the lateral hypothalamus or the prefrontal cortex

\begin{tabular}{|c|c|c|c|c|}
\hline & $\begin{array}{l}\text { Cocaine- } \\
\text { stimulated }\end{array}$ & $\begin{array}{l}\text { Cocaine } \\
\text { sham-stimulated }\end{array}$ & $\begin{array}{l}\text { Saline- } \\
\text { stimulated }\end{array}$ & $\begin{array}{l}\text { Saline } \\
\text { sham-stimulated }\end{array}$ \\
\hline \multicolumn{5}{|c|}{ NR1 in LH-treated rats } \\
\hline $\mathrm{aNAC}$ & $47 \pm 6$ & $67 \pm 5^{b}$ & $69 \pm 19$ & $100 \pm 19$ \\
\hline $\mathrm{pNAc}$ & $160 \pm 15$ & $132 \pm 13$ & $97 \pm 28$ & $100 \pm 25$ \\
\hline aVTA & $119 \pm 19$ & $80 \pm 7$ & $87 \pm 9$ & $100 \pm 9$ \\
\hline pVTA & $132 \pm 6$ & $142 \pm 10^{b}$ & $99 \pm 16$ & $100 \pm 14$ \\
\hline \multicolumn{5}{|c|}{ NR1 in PFC-treated rats } \\
\hline aNAc & $156 \pm 42^{a}$ & $65 \pm 15^{b}$ & $53 \pm 16^{c}$ & $100 \pm 25$ \\
\hline pNAc & $98 \pm 38^{a}$ & $48 \pm 10^{b}$ & $98 \pm 36$ & $100 \pm 27$ \\
\hline aVTA & $95 \pm 49$ & $78 \pm 26$ & $151 \pm 46$ & $100 \pm 21$ \\
\hline pVTA & $181 \pm 87$ & $134 \pm 36$ & $191 \pm 59$ & $100 \pm 20$ \\
\hline
\end{tabular}

Rats were perfused immediately after the saline challenge. Data are calculated from the mean DAB intensity \pm SEM from each subregion of each experimental group.

cocaine challenge. PFC-ICES, however, did not have a consistent effect on the response to drug-associated cues. Although it attenuated the ability of cocaine-associated cues to maintain drugseeking behavior under extinction conditions, PFC-ICES did not affect conditioned locomotion after a saline challenge. The reduced breaking point and lower number of lever presses in the PR test can be interpreted as a reduction in the reward value of cocaine or in the motivation to work for cocaine reward (Stafford et al., 1998). This effect, in turn, would reduce cue-induced cocaine craving and attenuate cocaine-seeking behavior under drug-free conditions, but might not affect conditioned locomotor responses.

A possible reason for the intriguing opposite effects of LHICES on locomotor hyperactivity after cocaine or saline challenge is the development of ICES-induced sensitization of the response to direct effects of the drug versus desensitization of the response to cocaine-associated cues. Cocaine-associated cues are critical for maintaining cocaine self-administration (Arroyo et al., 1998). Thus, the lack of effect on the cocaine PR test in LH-stimulated rats might be attributable to the opposing effects of the simultaneous exposure to the drug and the drug-associated cues. PFCICES, however, seems to attenuate both the direct effects of cocaine on behavior and the habitual, automatic (Tiffany, 1990; Tiffany and Carter, 1998) drug-conditioned behavior, without affecting cue-induced locomotion.
Because PFC-ICES had more promising therapeutic effects on cocaine-related behaviors, we further examined stimulation properties in this site using a lower stimulation frequency, and tested the longer-term effects of ICES treatment. The lower frequency was slightly (but not significantly) less effective in the short-term measurements but slightly more effective in the long-term PR tests. Overall, the effect of the $20 \mathrm{~Hz}$ dose was quite similar to that of the $100 \mathrm{~Hz}$, indicating that lower frequencies, which are safer and more feasible in TMS studies (Anderson et al., 2006), can be used in translational human studies. In brain stimulation reward studies, where animals control the rate of selfstimulation, higher-frequency stimulation reflects higher value of reward (Wise, 1996), and $20 \mathrm{~Hz}$ stimulation is not sufficient to induce reliable PFC selfstimulation (Schenk et al., 1985). Therefore, the significant effect of repeated 20 $\mathrm{Hz}$ ICES on cocaine seeking and consumption suggests that the relevant neuroadaptations induced by such treatment are not necessarily those associated with brain stimulation reward.

The effects of ICES on glutamatergic receptor subtypes are an initial step toward characterization of the neurochemical alterations induced by such treatment approach. These measurements revealed interesting contrasts between the effect of repeated cocaine administration and ICES treatment. The most pronounced effect of LH-ICES was in the VTA. ICES reduced GluR1 levels in the aVTA, in direct contrast to the upregulated GluR1 levels in this region caused by repeated cocaine infusions. This is consistent with previous findings showing that GluR1 levels in the VTA are transiently upregulated by repeated cocaine intake (Churchill et al., 1999). Furthermore, the downregulated GluR1 expression in the aVTA induced by ICES treatment is consistent with the decreased expression of GluR1 in the VTA after hypothalamic self-stimulation (Carlezon et al., 2001). Moreover, the specificity of the reduced GluR1 levels only in the aVTA, rather than the entire VTA, correlates with the attenuated response to drug-associated cues, as observed in our behavioral paradigms, and seems consistent to the finding that overexpression of GluR1 in the aVTA, but not pVTA, enhanced morphine place conditioning (Carlezon et al., 2000). Given that ICES treatment reversed the increase in GluR1 levels in the aVTA mediated by repeated cocaine administration, it is possible that this effect underlies the lower conditioned locomotion and drugseeking behavior observed in the LH-ICES group. On the other hand, the sensitized response to cocaine after LH-ICES was not correlated with any of the neurochemical measures in this study.

PFC-ICES did not alter the VTA glutamate receptor subtype levels, which is consistent with the lack of effect in the conditioned locomotion experiment. It did, however, affect the NAc. Repeated cocaine exposure reduced GluR1 levels in the pNAc and in the aNAc (core and shell) of cocaine-pretreated rats. This is consistent with previous reports that repeated cocaine exposure 

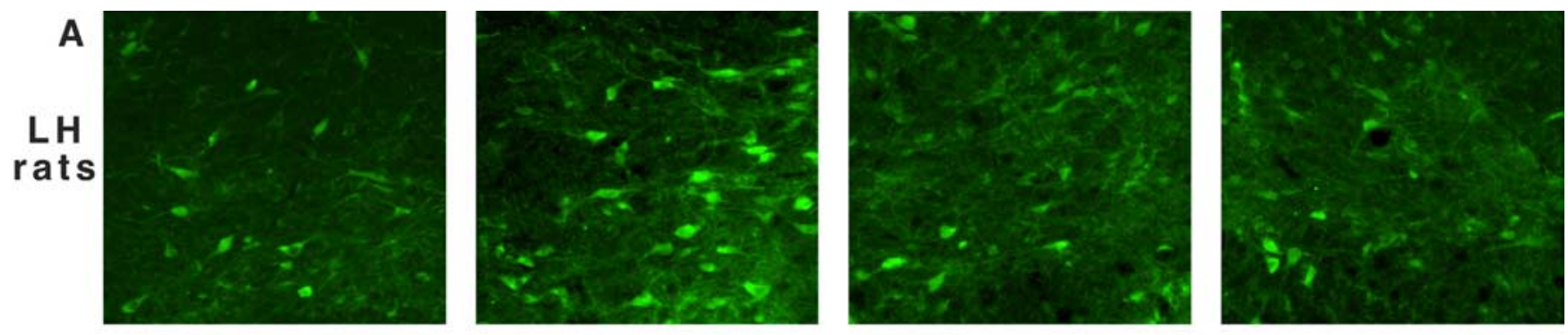

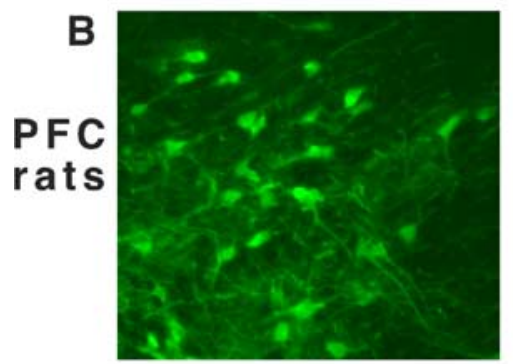

Cocaine-pretreated stimulated

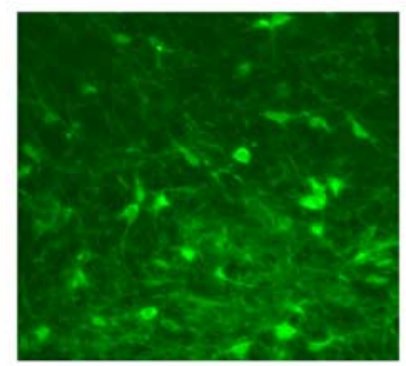
Cocaine-pretreated
sham-stimulated

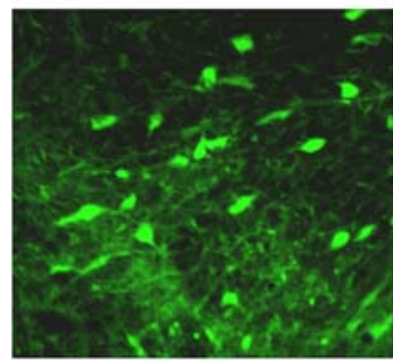

Saline-pretreated stimulated

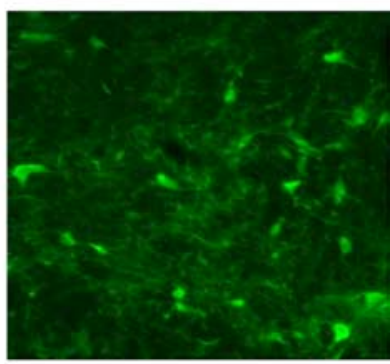

Saline-pretreated sham-stimulated

Figure 7. The effect of repeated cocaine and ICES on GluR1 in the anterior VTA. Representative immunohistochemical images of the anterior VTA from LH (A) and PFC (B) rats. For detailed quantification, see Table 1.

reduced GluR1 levels in the NAc (Ghasemzadeh et al., 1999; Lu et al., 2003). In addition, we demonstrated that PFC-ICES upregulated GluR1 levels in the aNAc core and shell. The findings regarding the aNAc shell are strikingly similar to those demonstrating that extinction training during withdrawal from chronic cocaine self-administration increased GluR1 levels in the NAc shell (Sutton et al., 2003). Recently, it was reported that increased GluR1 expression in the NAc shell is associated with decreased reward value of ICSS (Todtenkopf et al., 2006). Therefore, it is possible that PFC-ICES, partly through upregulation of GluR1 in the aNAc shell, attenuated the response to cocaine by activating brain circuits mediating extinction-like behaviors or by reducing the reward value of cocaine. Clearly, adaptations in the glutamate transmission, as demonstrated here, do not offer a full explanation for the behavioral effects observed in ICES-treated rats, and do not preclude contributions from additional neuronal systems.

The effects of ICES treatment on cocaine-seeking and cocaine self-administration were not caused by a general "impairment" in the reward system, as no changes were observed in the seeking or consumption of sucrose, a natural reward. Although they probably overlap to a large extent, the neuronal circuitries of natural and drug-related rewards are not identical (Carelli, 2002). Perhaps, the underlying neurocircuitry of natural rewards that are fundamental for survival is less prone to long-term manipulations and to disruptions mediated by ICES treatment.

Our findings for the sucrose self-administration groups, together with the lack of differences between stimulated and shamstimulated cocaine self-administration groups in the MWM measurements, indicate that the ICES-mediated effects on behavior are not caused by a general ICES-induced learning and memory deficit. It could also be argued that ICES treatment induced a general decrease in activity. This is unlikely, however, because an ICES-mediated reduction in locomotor activity occurred only in the postinjection phases and not during habituation. Further, in a general activity test, the behavior of the stimulated rats in the self-administration experiments did not differ from that of shamstimulated rats, and no differences were observed between stimulated and sham-stimulated sucrose self-administration groups.

The present findings demonstrate the potential usefulness of localized electrical stimulation in the treatment of addictive behaviors. Further experiments to explore the effects of ICES in different stimulation regimens and in other reward-related areas are needed. PFC-ICES might be more effective for treating addiction than LH-ICES because of its ability to reduce the response to the drug and the motivation to consume it (whereas stimulation of the LH might cause sensitization of the response). Nonetheless, the effect of $100 \mathrm{~Hz}$ PFC-ICES on motivation for cocaine intake began to dissipate, whereas lower (and safer) frequency stimulation lasted at least $10 \mathrm{~d}$ after treatment. Intracranial electrode implantation, however, is a drastic intervention with considerable risks. Repeated (nonchronic) deep TMS might serve as an alternative noninvasive treatment for neurological and psychiatric diseases (Zangen et al., 2005). Testing the efficacy of deep TMS as a treatment for addiction might offer a valuable tool for the treatment of this complex disorder.

\section{References}

Anderson B, Mishory A, Nahas Z, Borckardt JJ, Yamanaka K, Rastogi K, George MS (2006) Tolerability and safety of high daily doses of repetitive transcranial magnetic stimulation in healthy young men. J Ect 22:49-53.

Arroyo M, Markou A, Robbins TW, Everitt BJ (1998) Acquisition, maintenance and reinstatement of intravenous cocaine self-administration under a second-order schedule of reinforcement in rats: effects of conditioned cues and continuous access to cocaine. Psychopharmacology (Berl) 140:331-344.

Arvanitogiannis A, Tzschentke TM, Riscaldino L, Wise RA, Shizgal P (2000) Fos expression following self-stimulation of the medial prefrontal cortex. Behav Brain Res 107:123-132.

Bauco P, Wise RA (1997) Synergistic effects of cocaine with lateral hypothalamic brain stimulation reward: lack of tolerance or sensitization. J Pharmacol Exp Ther 283:1160-1167. 
Ben-Shahar O, Ettenberg A (1994) Repeated stimulation of the ventral tegmental area sensitizes the hyperlocomotor response to amphetamine. Pharmacol Biochem Behav 48:1005-1009.

Boudreau AC, Wolf ME (2005) Behavioral sensitization to cocaine is associated with increased AMPA receptor surface expression in the nucleus accumbens. J Neurosci 25:9144-9151.

Carelli RM (2002) Nucleus accumbens cell firing during goal-directed behaviors for cocaine vs. 'natural' reinforcement. Physiol Behav 76:379-387.

Carlezon Jr WA, Haile CN, Coppersmith R, Hayashi Y, Malinow R, Neve RL, Nestler EJ (2000) Distinct sites of opiate reward and aversion within the midbrain identified using a herpes simplex virus vector expressing GluR1. J Neurosci 20:RC62(1-5).

Carlezon Jr WA, Todtenkopf MS, McPhie DL, Pimentel P, Pliakas AM, Stellar JR, Trzcinska M (2001) Repeated exposure to rewarding brain stimulation downregulates GluR1 expression in the ventral tegmental area. Neuropsychopharmacology 25:234-241.

Chefer VI, Shippenberg TS (2002) Changes in basal and cocaine-evoked extracellular dopamine uptake and release in the rat nucleus accumbens during early abstinence from cocaine: quantitative determination under transient conditions. Neuroscience 112:907-919.

Churchill L, Swanson CJ, Urbina M, Kalivas PW (1999) Repeated cocaine alters glutamate receptor subunit levels in the nucleus accumbens and ventral tegmental area of rats that develop behavioral sensitization. J Neurochem 72:2397-2403.

Cornish JL, Kalivas PW (2000) Glutamate transmission in the nucleus accumbens mediates relapse in cocaine addiction. J Neurosci 20:1-5.

Ghasemzadeh MB, Nelson LC, Lu XY, Kalivas PW (1999) Neuroadaptations in ionotropic and metabotropic glutamate receptor mRNA produced by cocaine treatment. J Neurochem 72:157-165.

Hand TH, Franklin KB (1983) The influence of amphetamine on preference for lateral hypothalamic versus prefrontal cortex or ventral tegmental area self-stimulation. Pharmacol Biochem Behav 18:695-699.

Hyman SE, Malenka RC (2001) Addiction and the brain: the neurobiology of compulsion and its persistence. Nat Rev Neurosci 2:695-703.

Hyman SE, Malenka RC, Nestler EJ (2006) Neural mechanisms of addiction: the role of reward-related learning and memory. Annu Rev Neurosci 29:565-598.

Kalivas PW, Volkow ND (2005) The neural basis of addiction: a pathology of motivation and choice. Am J Psychiatry 162:1403-1413.

Leshner AI (1997) Addiction is a brain disease, and it matters. Science 278:45-47.

Lu L, Grimm JW, Shaham Y, Hope BT (2003) Molecular neuroadaptations in the accumbens and ventral tegmental area during the first 90 days of forced abstinence from cocaine self-administration in rats. J Neurochem 85:1604-1613.

Nestler EJ (2001) Molecular basis of long-term plasticity underlying addiction. Nat Rev Neurosci 2:119-128.

Nestler EJ (2002) From neurobiology to treatment: progress against addiction. Nat Neurosci 5 [Suppl]:1076-1079.

Paxinos G, Watson C (1998) The rat brain in stereotaxic coordinates, Ed 4. San Diego: Academic.

Schenk S, Shizgal P (1982) The substrates for lateral hypothalamic and medial pre-frontal cortex self-stimulation have different refractory periods and show poor spatial summation. Physiol Behav 28:133-138.

Schenk S, Snow S (1994) Sensitization to cocaine's motor activating prop- erties produced by electrical kindling of the medial prefrontal cortex but not of the hippocampus. Brain Res 659:17-22.

Schenk S, Prince C, Shizgal P (1985) Spatio-temporal integration in the substrate for self-stimulation of the prefrontal cortex. Physiol Behav 35:303-306.

Sesack SR, Pickel VM (1992) Prefrontal cortical efferents in the rat synapse on unlabeled neuronal targets of catecholamine terminals in the nucleus accumbens septi and on dopamine neurons in the ventral tegmental area. J Comp Neurol 320:145-160.

Stafford D, LeSage MG, Glowa JR (1998) Progressive-ratio schedules of drug delivery in the analysis of drug self-administration: a review. Psychopharmacology (Berl) 139:169-184.

Sutton MA, Schmidt EF, Choi KH, Schad CA, Whisler K, Simmons D, Karanian DA, Monteggia LM, Neve RL, Self DW (2003) Extinction-induced upregulation in AMPA receptors reduces cocaine-seeking behaviour. Nature 421:70-75.

Tiffany ST (1990) A cognitive model of drug urges and drug-use behavior: role of automatic and nonautomatic processes. Psychol Rev 97:147-168.

Tiffany ST, Carter BL (1998) Is craving the source of compulsive drug use? J Psychopharmacol 12:23-30.

Todtenkopf MS, Parsegian A, Naydenov A, Neve RL, Konradi C, Carlezon Jr WA (2006) Brain reward regulated by AMPA receptor subunits in nucleus accumbens shell. J Neurosci 26:11665-11669.

Vanderschuren LJ, Kalivas PW (2000) Alterations in dopaminergic and glutamatergic transmission in the induction and expression of behavioral sensitization: a critical review of preclinical studies. Psychopharmacology (Berl) 151:99-120.

Vorel SR, Liu X, Hayes RJ, Spector JA, Gardner EL (2001) Relapse to cocaine-seeking after hippocampal theta burst stimulation. Science 292:1175-1178.

Wise RA (1996) Addictive drugs and brain stimulation reward. Annu Rev Neurosci 19:319-340.

Wise RA, Munn E (1993) Effects of repeated amphetamine injections on lateral hypothalamic brain stimulation reward and subsequent locomotion. Behav Brain Res 55:195-201.

Wolf ME (1998) The role of excitatory amino acids in behavioral sensitization to psychomotor stimulants. Prog Neurobiol 54:679-720.

Wolf ME, Sun X, Mangiavacchi S, Chao SZ (2004) Psychomotor stimulants and neuronal plasticity. Neuropharmacology 47 [Suppl] 1:61-79.

Yadin E, Guarini V, Gallistel CR (1983) Unilaterally activated systems in rats self-stimulating at sites in the medial forebrain bundle, medial prefrontal cortex, or locus coeruleus. Brain Res 266:39-50.

You ZB, Tzschentke TM, Brodin E, Wise RA (1998) Electrical stimulation of the prefrontal cortex increases cholecystokinin, glutamate, and dopamine release in the nucleus accumbens: an in vivo microdialysis study in freely moving rats. J Neurosci 18:6492-6500.

You ZB, Chen YQ, Wise RA (2001) Dopamine and glutamate release in the nucleus accumbens and ventral tegmental area of rat following lateral hypothalamic self-stimulation. Neuroscience 107:629-639.

Zangen A, Shalev U (2003) Nucleus accumbens beta-endorphin levels are not elevated by brain stimulation reward but do increase with extinction. Eur J Neurosci 17:1067-1072.

Zangen A, Roth Y, Voller B, Hallett M (2005) Transcranial magnetic stimulation of deep brain regions: evidence for efficacy of the H-coil. Clin Neurophysiol 116:775-779. 